\title{
MIXED SPECTRAL TYPES FOR THE ONE-FREQUENCY DISCRETE QUASI-PERIODIC SCHRÖDINGER OPERATOR
}

\author{
SHIWEN ZHANG
}

(Communicated by Michael Hitrik)

\begin{abstract}
We consider a family of one-frequency discrete analytic quasiperiodic Schrödinger operators. We show that this family provides an example of coexistence of absolutely continuous and point spectrum for some parameters as well as coexistence of absolutely continuous and singular continuous spectrum for some other parameters.
\end{abstract}

\section{INTRODUCTION}

In Bjer, Bjerklöv considers the following discrete quasi-periodic Schrödinger operator on $l^{2}(\mathbb{Z})$ :

$$
\left(H_{K, \theta, \omega} u\right)_{n}=-u_{n+1}-u_{n-1}+V(\theta+n \omega) u_{n}, \quad n \in \mathbb{Z},
$$

where

$$
V(\theta)=\exp (K f(\theta+\omega))+\exp (-K f(\theta)),
$$

$\theta, \omega \in \mathbb{T}^{b}, f: \mathbb{T}^{b} \rightarrow \mathbb{R}$ is assumed to be a non-constant real-analytic function with zero mean, $\int_{\mathbb{T}^{b}} f(\theta) \mathrm{d} \theta=0$ and $K \in \mathbb{R}$ is any constant. Consider the Lyapunov exponent $L(E)$ (see the next section). In this explicit example, Bjerklöv shows that for large $K$ we have a situation with mixed dynamics: zero Lyapunov exponent in a region close to $E=0$ and positive for larger $E$.

In this paper, we are going to show that for the one-frequency case, in Bjerklöv's example (1.1), mixed dynamics actually lead to mixed spectra: for some parameters $(\theta, \omega), H_{K, \theta, \omega}$ has mixed absolutely continuous and point spectrum, and for some other $(\theta, \omega), H_{K, \theta, \omega}$ has mixed absolutely continuous and singular continuous spectrum.

Without loss of generality, we assume that $\|f\|_{C^{1}(\mathbb{T})}=1$ and $f$ has analytic extension to the strip $|\operatorname{Im} z|<h$, where $h \gg K$ (e.g., $f$ can be taken as any entire function). It follows from Bjer that $\min \left\{E \in \sigma\left(H_{K, \theta, \omega}\right)\right\}=0$ for any $K, \theta, \omega$. And also it is not hard to show that $\max \left\{E \in \sigma\left(H_{K, \theta, \omega}\right)=e^{K\|f\|_{\infty}}\right.$. For any $\epsilon>0$ small (w.l.o.g. we assume $0<\epsilon<1$ ), denote $I_{\epsilon, K}=\left[\epsilon, 4 e^{K\|f\|_{\infty}}\right]$. We have $I_{\epsilon, K} \cap \sigma\left(H_{K, \theta, \omega}\right) \neq \emptyset$. We say the frequency $\omega \in \mathbb{T}$ satisfies the Diophantine

Received by the editors April 24, 2015 and, in revised form, August 5, 2015.

2010 Mathematics Subject Classification. Primary 37A30, 47B36, 82B44.

Key words and phrases. Quasi-periodic Schrödinger operators, mixed spectral types, Lyapunov exponent, almost reducibility.

This research was partially supported by NSF DMS-1401204. 
Condition (denoted by $\omega \in D C(\kappa, \tau)$ ) if

$$
\|\omega \cdot n\| \geq \frac{\kappa}{|n|^{\tau}}, \quad \forall n \in \mathbb{Z} \backslash\{0\},
$$

for some $\kappa>0, \tau>0$. We say $\omega$ satisfies the Strong Diophantine Condition (denoted by $\omega \in S D C(\kappa)$ ) if

$$
\|\omega \cdot n\| \geq \frac{\kappa}{|n|(\log (|n|+1))^{2}}, \quad \forall n \in \mathbb{Z} \backslash\{0\},
$$

for some $\kappa>0$. Denote $D C=\bigcup_{\kappa} D C(\kappa, \tau)$ for some fixed $\tau>1$ and $S D C=$ $\bigcup_{\kappa} S D C(\kappa)$. It is well known that $D C \supseteq S D C$ and both of them have full Lebesgue measure 1

The main results are as follows.

Theorem 1. Let $V$ be given as in (1.2). Fix $\omega_{0} \in D C(\kappa, \tau)$. For any $\epsilon>0$, there are $K=K\left(\omega_{0}, \epsilon, f\right)>0, \delta=\delta\left(\omega_{0}, \epsilon, K\right)>0$, and for any $\omega \in B_{\delta}\left(\omega_{0}\right):=\{\omega \in \mathbb{T}$ : $\left.\left|\omega-\omega_{0}\right|<\delta\right\}$, there is $0<\epsilon_{0}=\epsilon_{0}\left(\omega, K, h,\|f\|_{h}\right)<\epsilon$ such that:

(a) For a.e. $\omega \in B_{\delta}\left(\omega_{0}\right)$ and a.e. $\theta \in \mathbb{T}, H_{K, \theta, \omega}$ has pure point spectrum in $I_{\epsilon, K}$ with exponentially decaying eigenvectors and has purely absolutely continuous spectrum in $\left[0, \epsilon_{0}\right]$.

(b) For a.e. $\omega \in B_{\delta}\left(\omega_{0}\right)$, there is a dense $G_{\delta}$ set of $\theta$, such that $H_{K, \theta, \omega}$ has purely singular continuous spectrum in $I_{\epsilon, K}$ and has purely absolutely continuous spectrum in $\left[0, \epsilon_{0}\right]$.

(c) For $\omega$ in a dense subset of $B_{\delta}\left(\omega_{0}\right)$ and for any $\theta, H_{K, \theta, \omega}$ has purely singular continuous spectrum in $I_{\epsilon, K}$ and has purely absolutely continuous spectrum in $\left[0, \epsilon_{0}\right]$.

Previously, Bourgain [Bo] constructed a quasi-periodic operator with two frequencies which has coexistence of absolutely continuous and point spectrum. While mixed spectra are expected to occur for generic one-frequency operators, such examples for the discrete case have been considered difficult to construct explicitly. Recently Bjerklöv and Krikorian [BK announced an example of this nature. Avila in Theorem 13 of A3 showed that in the neighborhood of the critical almost Mathieu operator, there are operators with at least $n$ alternances between the subcritical and supercritical regimes for any $n$. Such operators are strong potential candidates for coexistence of p.p./s.c. and a.c. spectrum (with, moreover, many alternances). For the continuous model, Fedotov and Klopp [FK] showed coexistence of absolutely continuous and singular spectrum for a family of quasi-periodic operators and also gave a criterion for the existence of absolutely continuous and singular spectrum in the semi-classical regime.

Here we give a short proof which shows that the operator (1.1) with potential (1.2) has mixed spectral types. The mixed nature of the spectrum follows from the coexistence of the positive Lyapunov exponent and the zero Lyapunov exponent which was obtained in Bjer and a combination of several recent results on localization, reducibility and continuity.

\footnotetext{
${ }^{1}$ Here $\|\cdot\|$ means the distance to the closest integer.
} 


\section{Singular spectrum in the positive Lyapunov exponent Region}

Denote

$$
A(\theta, E)=\left(\begin{array}{cc}
V(\theta)-E & -1 \\
1 & 0
\end{array}\right), \theta \in \mathbb{T}, \quad A^{n}(\theta, E)=\prod_{k=n-1}^{0} A(\theta+k \omega, E), n>0 .
$$

The Lyapunov exponent as usual (see [CFKS] is defined by

$$
L(E)=\lim _{n \rightarrow \infty} \int_{\mathbb{T}} \frac{1}{n} \log \left\|A^{n}(\theta, E)\right\| \mathrm{d} \theta \geq 0 .
$$

In the following, we would like to fix $f$ and consider the Lyapunov exponent $L(E, \omega, K)$ as the function of energy $E$, frequency $\omega$, and parameter $K$. In Bjer, Bjerklöv proved that:

Theorem 2 ( $(\mathrm{Bjer})$. Assume that $V$ is as in (1.2), and that $\omega \in D C(\kappa, \tau)$. Then for any $\epsilon>0$ there is a $K_{0}=K_{0}(\epsilon, f, \kappa, \tau)>0$ and $c=c(f)>0$ such that for all $K>K_{0}$, we have

$$
L(E, \omega, K) \geq c K, \text { for all } E \notin[0, \epsilon] .
$$

The proof is based on the Large Deviation Theorem (LDT)-Avalanche Principle (AvP) scheme developed by Bourgain, Goldstein, and Schlag [BG, GS1]. Due to some technical reasons, the largeness of $K$ depends on the Diophantine Conditions of $\omega$ in this theorem, which means the positivity is not uniform for all $\omega$. However, we can get the following local non-perturbative positivity. Bourgain and Jitomirskaya showed that the Lyapunov exponent is jointly continuous in $(\omega, E)$ at any irrational frequency (Theorem 1, [BJ]). The following result is obvious:

Proposition 1. Fix any $\epsilon>0$ and $\omega_{0} \in D C(\kappa, \tau)$, let $K_{0}=K_{0}\left(\epsilon, f, \omega_{0}\right)>0$ be given as in Theorem 2. Then for any $K>K_{0}$, there is $\delta=\delta\left(\omega_{0}, \epsilon, K\right)>0$, such that for any $\omega \in B_{\delta}\left(\omega_{0}\right), L(E, \omega, K)>0$ on $I_{\epsilon, K}$, where the lower bound only depends on $\omega_{0}, \epsilon, K, f$ and is uniform in $E$ and $\omega$.

The absence of a.c. spectrum on $I_{\epsilon, K}$ is therefore obvious due to Kotani theory; see $[\mathrm{K}]$ and the discrete version in $[\underline{\mathrm{S}}$. What we want to claim is the pure point spectrum or purely singular continuous spectrum in this region.

Anderson Localization (part (a)): Let $\Omega=S D C \cap B_{\delta}\left(\omega_{0}\right)$, which is a full measure subset of $B_{\delta}\left(\omega_{0}\right)$. Notice that the positivity of $L(E, \omega, K)$ is uniform for $E \in I_{\epsilon, K}$ and $\omega \in \Omega$. Then according to the non-perturbative localization result of Bourgain and Goldstein (see Theorem 10.1 and Remark (3), Chapter 10 in Bo1] ), for any $\theta \in \mathbb{T}$, a.e. $\omega \in \Omega, H_{K, \theta, \omega}$ exhibits A.L. in $I_{\epsilon, K}$, i.e., $H_{K, \theta, \omega}$ has pure point spectrum restricted in $I_{\epsilon, K}$ and the corresponding eigenfunctions decay exponentially. Thus by Fubini's theorem, $H_{K, f, \theta, \omega}$ has A.L. for a.e. $\omega \in \Omega$ and a.e. $\theta \in \mathbb{T}$.

Purely s.c. spectrum (part (b)): Let $\Omega$ be the same as in the previous part. Goldstein and Schlag [GS2] show that for a.e. $\omega \in \Omega$, the intersection $\sigma\left(H_{K, f, \theta, \omega}\right) \cap I_{\epsilon, K}$ is a Cantor set (see Theorem 1.1 in GS2]). Then according to a theorem of Gordon G2], nowhere dense structure of the spectrum implies the absence of point spectrum for a dense $G_{\delta}$ set of $\theta$ (see Theorem 6 in [G2]). Therefore, for a.e. $\omega \in \Omega$, there is a dense $G_{\delta}$ set of $\theta$ such that $H_{K, \theta, \omega}$ has purely singular continuous spectrum in $I_{\epsilon, K}$. 
Purely s.c. spectrum (part (c)): Absence of point spectrum in this part is based on rational approximation. More precisely, denote

$$
\beta(\omega):=\limsup _{n} \frac{\log q_{n+1}}{q_{n}}
$$

where $\frac{p_{n}}{q_{n}}$ is the $n^{\text {th }}$ rational approximation of $\omega$. Notice that

$$
\sup _{n} \sup _{(\theta, \omega) \in \mathbb{T}^{2}, E \in I_{\epsilon, K}} \frac{1}{|n|} \log \left\|A^{n}(\theta, E)\right\| \leq 10 K .
$$

Then by a standard Gordon type argument (see e.g. G1, CFKS]), if $\beta(\omega)>$ $40 K$, then for any $\theta, H_{K, \theta, \omega}$ does not have any point spectrum. Combined with the positivity of the Lyapunov exponent in $I_{\epsilon, K}$, the proof for the purely s.c. spectrum of part (c) is completed. Notice that for any $\beta_{0} \in$ $[0, \infty]$, the level set $\Omega_{\beta_{0}}:=\left\{\omega: \beta(\omega)=\beta_{0}\right\}$ is a dense set. For later purpose, we would like to pick the dense subset $\Omega_{\beta_{0}} \cap B_{\delta}\left(\omega_{0}\right)$ with $40 K<\beta_{0}<h / 2$.

\section{Absolutely continuous spectrum near the Bottom}

Next we are going to show that for any $\omega \in \mathbb{T}$ with finite $\beta(\omega)$, if the energy $E$ is sufficiently small (depends on $\omega$ ), then the Schrödinger cocycle is almost reducible. This will imply purely a.c. spectrum near the bottom of the spectrum for any phase $\theta$. To complete the proof of the main theorem, we first pick some $\omega$ near $\omega_{0}$ and some $\theta$ which will give us point spectrum or singular continuous spectrum as in the previous part. Then for these pairs of $(\omega, \theta)$, we apply the almost reducibility result to get the coexistence of two types of spectrum.

The key step to find purely a.c. spectrum near the bottom is the following reducibility result at $E=0$, which generalizes Lemma 5.1 in $B$ Bjer to the case $0<\beta(\omega)<\infty$.

Proposition 2. For any frequency $\omega$ with $\beta(\omega)<\infty$, if $h>2 \beta$, then there exists an analytic transformation $C: \mathbb{T} \rightarrow S L(2, \mathbb{R})$ such that

$$
C(\theta+\omega) A(\theta, 0) C(\theta)^{-1}=A_{0},
$$

where

$$
A_{0}=\left(\begin{array}{cc}
1 & \hat{k} \\
0 & 1
\end{array}\right), \quad \hat{k} \in \mathbb{R}
$$

Remark 3.1. If $\omega$ is Diophantine or $\beta=0$, the proposition has been proved in Lemma 5.1 of $\mathrm{Bjer}$. If $\beta>0$, we can still find such a transformation $C$ provided $h$ is large. The only loss is the decrease of the width of the analytic strip. Also the analytic norm of the transformation and the constant could be very large. Actually, $C$ has an analytic extension to the strip $|\operatorname{Im} z|<h-2 \beta$, with $\|C\|_{h-2 \beta} \sim e^{K\|f\|_{h}}$. We also have $|\hat{k}| \sim e^{K\|f\|_{h}}$.

Proof. Recall the main steps in the proof of Lemma 5.1 in Bjer, if there are $g, h: \mathbb{T} \rightarrow \mathbb{R}$ satisfying the equations

$$
\begin{aligned}
g(\theta+\omega)-g(\theta) & =f(\theta+\omega), \\
k(\theta)=-e^{-K g(\theta-\omega)-K g(\theta)} & , \quad \hat{k}=\int_{\mathbb{T}} k(\theta) \mathrm{d} \theta, \\
h(\theta+\omega)-h(\theta) & =\hat{k}-k(\theta),
\end{aligned}
$$


then set

$$
C(\theta)=\left(\begin{array}{cc}
1 & h(\theta) \\
0 & 1
\end{array}\right) \cdot\left(\begin{array}{cc}
0 & \exp (-K g(\theta-\omega)) \\
-\exp (K g(\theta-\omega)) & \exp (K g(\theta))
\end{array}\right) \in S L(2, \mathbb{R}) .
$$

Direct computation shows that

$$
C(\theta+\omega) A(\theta, 0) C(\theta)^{-1}=\left(\begin{array}{cc}
1 & \hat{k} \\
0 & 1
\end{array}\right),
$$

which is the desired form.

For real analytic $f$ with zero average, if $\omega$ is Diophantine, equations (3.1), (3.2) always have real analytic solutions $g, h$, which is the case in Bjer.

If $\beta>0$, recall for $f$ analytic in the strip $|\operatorname{Im} z|<h$ that the Fourier coefficients of $f$ satisfy $\left|\hat{f}_{k}\right| \leq\|f\|_{h} e^{-h|k|}$, therefore, from Fourier series expansion, equation (3.1) has an analytic solution $g$ in the strip $|\operatorname{Im} z|<h-\beta$ provided $h>\beta$. From the definition of $k, k(\theta)$ also has analytic extension to the strip $|\operatorname{Im} z|<h-\beta$ with $\|k\|_{h-\beta} \sim e^{K\|g\|_{h-\beta}} \sim e^{K\|f\|_{h}}$. Then for the same reason, equation (3.2) also has an analytic solution $h$ in the strip $|\operatorname{Im} z|<h-2 \beta$ provided $h-\beta>\beta$.

Then it is easy to see that by applying $C$ to $A(\theta, E)$, we have

$$
\begin{aligned}
C(\theta+\omega) A(\theta, E) C(\theta)^{-1} & =A_{0}+C(\theta+\omega)\left(\begin{array}{cc}
-E & 0 \\
0 & 0
\end{array}\right) C(\theta)^{-1} \\
& =A_{0}+E F(\theta) \in S L(2, \mathbb{R})
\end{aligned}
$$

where

$$
F(\theta)=C(\theta+\omega)\left(\begin{array}{cc}
-1 & 0 \\
0 & 0
\end{array}\right) C(\theta)^{-1} .
$$

From the proof of Proposition 2, we see that $F$ has analytic extension to the strip $|I m z|<h-2 \beta$ and the largeness of $\left\|A_{0}\right\|,\|F\|_{h-2 \beta}$ depends on $\omega, K,\|f\|_{h}$.

For Diophantine frequency, as Bjerklöv mentioned in Remark 2 in Bjer, one can show purely absolutely continuous spectrum for sufficiently small $E$ based on the KAM approach as in E. Here since we also need to deal with Liouvillean frequency, we want to prove all cases together with the almost reducibility concept.

We say the skew product system $(\omega, A)$ is almost reducible if there exist $\eta>0$ and a sequence of analytic maps $B^{(n)}: \mathbb{T} \rightarrow P S L(2 ; \mathbb{R})$, admitting holomorphic extensions to the common strip $|\operatorname{Im} z|<\eta$ such that $B^{(n)}(z+\omega) A(z) B^{(n)}(z)^{-1}$ converges to a constant uniformly in $|\operatorname{Im} z|<\eta$. We need the following result about almost reducibility:

Proposition 3 (Corollary 1.2, [A1]). Any one-frequency analytic quasi-periodic $S L(2, \mathbb{R})$ cocycle close to constant is analytically almost reducible.

Proof of purely a.c. spectrum near the bottom. According to Proposition 3 , ( $\omega, A_{0}+$ $E F(\theta)$ ) is almost reducible for small $E$. More precisely, consider $A_{0}+E F$ in (3.3). There exists $\epsilon_{0}=\epsilon_{0}\left(\omega,\left\|A_{0}\right\|, h,\|F\|_{h-2 \beta}\right)<\epsilon$ such that for $0<E<\epsilon_{0}$, $\left(\omega, A_{0}+E F(\theta)\right)$ is almost reducible. (Such a quantitative version can be found in Theorem 1.2 of [HY] and Corollary 1.3 of [YZ].) Therefore, $(\omega, A(\theta, E))$ is also 
almost reducible for $0<E<\epsilon_{0}$. As a corollary of almost reducibility [A1, A2, 2 we have that for any $\theta, H_{K, f, \theta, \omega}$ has purely absolutely continuous spectrum in $\left[0, \epsilon_{0}\right]$.

\section{ACKNOWLEDGEMENTS}

The author would like to thank Svetlana Jitomirskaya for many useful discussions. The author also would like to thank the Isaac Newton Institute for Mathematical Sciences, Cambridge, for support and hospitality during the Programme Periodic and Ergodic Spectral Problems, where work on this paper was undertaken.

\section{REFERENCES}

[A1] A. Avila, Almost reducibility and absolute continuity I, preprint.

[A2] A. Avila, Almost reducibility and absolute continuity II (in preparation).

[A3] A. Avila, Global theory of one-frequency Schrodinger operators II, preprint.

[Bo] J. Bourgain, On the spectrum of lattice Schrödinger operators with deterministic potential. II, J. Anal. Math. 88 (2002), 221-254, Dedicated to the memory of Tom Wolff, DOI 10.1007/BF02786578. MR1984594 (2004e:47046)

[Bo1] J. Bourgain, Green's function estimates for lattice Schrödinger operators and applications, Annals of Mathematics Studies, vol. 158, Princeton University Press, Princeton, NJ, 2005. MR2100420 (2005j:35184)

[BG] J. Bourgain and M. Goldstein, On nonperturbative localization with quasi-periodic potential, Ann. of Math. (2) 152 (2000), no. 3, 835-879, DOI 10.2307/2661356. MR.1815703 (2002h:39028)

[BJ] J. Bourgain and S. Jitomirskaya, Continuity of the Lyapunov exponent for quasiperiodic operators with analytic potential, J. Statist. Phys. 108 (2002), no. 5-6, 1203-1218, Dedicated to David Ruelle and Yasha Sinai on the occasion of their 65th birthdays, DOI 10.1023/A:1019751801035. MR1933451 (2004c:47073)

[Bjer] K. Bjerklöv, Explicit examples of arbitrarily large analytic ergodic potentials with zero Lyapunov exponent, Geom. Funct. Anal. 16 (2006), no. 6, 1183-1200, DOI 10.1007/s00039-006-0581-8. MR2276537 (2008b:47069)

[BK] K. Bjerklöv and R. Krikorian, Coexistence of ac and pp spectrum for quasiperiodic $1 \mathrm{D}$ Schrödinger operators (in preparation).

[CFKS] H. L. Cycon, R. G. Froese, W. Kirsch, and B. Simon, Schrödinger operators with application to quantum mechanics and global geometry, Springer Study Edition, Texts and Monographs in Physics, Springer-Verlag, Berlin, 1987. MR883643 (88g:35003)

[E] L. H. Eliasson, Floquet solutions for the 1-dimensional quasi-periodic Schrödinger equation, Comm. Math. Phys. 146 (1992), no. 3, 447-482. MR1167299 (93d:34141)

[FK] Alexander Fedotov and Frédéric Klopp, Coexistence of different spectral types for almost periodic Schrödinger equations in dimension one, Mathematical results in quantum mechanics (Prague, 1998), Oper. Theory Adv. Appl., vol. 108, Birkhäuser, Basel, 1999, pp. 243-251. MR.1708804 (2000g:34138)

[G1] A. Ja. Gordon, The point spectrum of the one-dimensional Schrödinger operator (Russian), Uspehi Mat. Nauk 31 (1976), no. 4(190), 257-258. MR0458247 (56 \#16450)

[G2] Alexander Y. Gordon, A spectral alternative for continuous families of self-adjoint operators, J. Spectr. Theory 3 (2013), no. 2, 129-145, DOI 10.4171/JST/40. MR3042762

[GS1] Michael Goldstein and Wilhelm Schlag, Hölder continuity of the integrated density of states for quasi-periodic Schrödinger equations and averages of shifts of subharmonic functions, Ann. of Math. (2) 154 (2001), no. 1, 155-203, DOI 10.2307/3062114. MR:1847592(2002h:82055)

\footnotetext{
${ }^{2}$ Corollary 1.6 in [A1] established absolutely continuous spectrum as a consequence of almost reducibility for a.e. $\theta$, thus completing the proof of part (a) and a.e. version of (c) of Theorem 1. Part (b) and extending part (c) to all $\theta$ depend on the "all $\theta$ " version of this statement, to appear in A2.
} 
[GS2] Michael Goldstein and Wilhelm Schlag, On resonances and the formation of gaps in the spectrum of quasi-periodic Schrödinger equations, Ann. of Math. (2) 173 (2011), no. 1, 337-475, DOI 10.4007/annals.2011.173.1.9. MR2753606 (2012d:81100)

[HY] Xuanji Hou and Jiangong You, Almost reducibility and non-perturbative reducibility of quasi-periodic linear systems, Invent. Math. 190 (2012), no. 1, 209-260, DOI 10.1007/s00222-012-0379-2. MR.2969277

[K] S. Kotani, Lyapunov indices determine absolutely continuous spectra of stationary random one-dimensional, Schrödinger operators, Proc. Kyoto Stoch. Conf., 1982.

[S] Barry Simon, Kotani theory for one-dimensional stochastic Jacobi matrices, Comm. Math. Phys. 89 (1983), no. 2, 227-234. MR709464 (85d:60122)

[YZ] Jiangong You and Qi Zhou, Embedding of analytic quasi-periodic cocycles into analytic quasi-periodic linear systems and its applications, Comm. Math. Phys. 323 (2013), no. 3, 975-1005, DOI 10.1007/s00220-013-1800-4. MR3106500

Department of Mathematics, University of California Irvine, Irvine, California 92617

E-mail address: shiwez1@uci.edu 